\title{
A formação da Agenda da Estratégia de Saúde da Família: Uma reflexão histórica em múltiplos fluxos.
}

\section{The formation of the Agenda of the Family Health strategy: An historical reflection in multiple streams.}

\section{La formación de la Agenda de la Estrategia de Salud familiar: Un reflexión histórica en corrientes múltiples.}

Juliana Mota LOUREIRO

Ana Valéria M. MENDONÇA ${ }^{2}$

Maria Paula do Amaral ZAITUNE ${ }^{2}$

Maria Fátima de SOUSA ${ }^{2}$

RESUMO: A Estratégia Saúde da Família é considerada uma das respostas à chamada crise do modelo assistencial enfrentada pelo Sistema Único de Saúde. Foi protagonizada por gestores, trabalhadores de saúde, pesquisadores e militantes deste sistema e implantada no Brasil em 1994. Este artigo teve por objetivo identificar os principais fluxos (problemas, político e soluções) que compõem o processo de formação da agenda da Estratégia Saúde da Família, no período de 1994 a 2011, a partir dos principais momentos estruturantes da Atenção Básica no Brasil. Utilizou-se o Modelo de Múltiplos Fluxos (Multiple Streams) de John Kingdon para analisar o processo de formação da agenda governamental e observou-se que os problemas prioritários da atenção básica, a exemplo do acesso e da integralidade, geram soluções que são definidas a partir da confluência de interesses de diversos atores e grupos políticos.

Palavras-chave: Atenção Básica à Saúde, Saúde da Família, Políticas Públicas e agenda de saúde.

\footnotetext{
ABSTRACT: Family Health Strategy, object of this study and implemented in Brazil since 1994, is considered one of the responses for the crisis faced by the National Health System through its managers, health workers, researchers and defensors of this System. The purpose of this paper was identify the main streams (problems, politics and policy) that are the elements of the process of the creation of the Family Health Strategy agenda, between the period of 1994 and 2011, as structured element of the Primary Health Care in Brazil through the application of Multiple Streams Model of John Kingdon to analyze the governmental agenda and it was observed that the solutions 1 Mestre em Saúde Coletiva pela Universidade de Brasília. Analista Técnico de Políticas Sociais no Ministério da Saúde do Brasil.jumloureiro@gmail.com 2 Docentes do Departamento de Saúde Coletiva - Faculdade de Ciências da Saúde - Universidade de Brasília.
} 
presented to address the priority problems of primary care: access and integrality are defined from the confluence of interests of various actors and political groups.

Key words: Primary Health Care, family health, public policies, Health Priority Agenda.

RESUMEN: La Estrategia de Salud de la Familia es considerada una de las respuestas a la llamada de la crisis que enfrenta el modelo de atención del Sistema de Salud. Se llevó a cabo por los administradores, trabajadores de salud, investigadores y activistas de este sistema e implementado en Brasil en 1994. Este artículo tuvo como objetivo identificar el flujo principal (problemas, soluciones y política) que componen el proceso de formación de la agenda de la Estrategia de Salud, de 1994 a 2011, de los principales momentos de estructuración de la Atención Primaria en Brasil. Se utilizó el modelo de flujos múltiples (múltiples flujos) John Kingdon para analizar el proceso de formación de la agenda del gobierno y se observó que los problemas prioritarios de atención primaria, como el acceso y la integridad, generan soluciones que se establecen para de la confluencia de intereses de los diversos actores y grupos políticos.

Palabras clave: Atención Primaria de la Salud, Salud, Políticas Públicas y la Agenda de Salud.

\section{INTRODUÇÃO}

Este artigo versa sobre a Estratégia de Saúde da Família - ESF, que foi uma medida governamental implantada no Brasil em 1994 frente à crise do modelo assistencial do Sistema Único de Saúde.

Utilizou-se de referenciais da Administração Pública, por envolver processos decisórios; e da Ciência Política, por envolver os poderes do Estado e dos governos, sendo ambas relacionadas às políticas de saúde, parte do campo teórico da Saúde Coletiva.

O Modelo de Múltiplos Fluxos (Multiple Streams) de John Kingdon ${ }^{1}$ foi usado para alcançar o objetivo do estudo, em que foi possível identificar os principais problemas, fatores políticos, fluxo de soluções de momentos que compuseram o processo de formação da agenda da ESF e, assim, possibilitar a melhor compreensão de como as prioridades ocuparam a agenda governamental.

Optou-se pelo corte temporal de 1994 a 2011, período este constituinte dos principais marcos históricos de implantação e implementação da ESF no Brasil.

\section{O modelo de análise: teoria dos múltiplos fluxos}

O modelo proposto por Kingdon ${ }^{1}$ se baseia no modelo de garbage can, criado por Cohen, March e Olsen ${ }^{2}$, no qual as decisões são descritas como emergentes de um processo em que a definição de atores, problemas, alternativas e outros aspectos são desenvolvidos simultaneamente e as escolhas são feitas com esses insumos, à vista de uma oportunidade de escolha. 
A Teoria dos Múltiplos Fluxos apresenta processos de decisão compostos por três fluxos (streams) dinâmicos, distintos e independentes; o fluxo dos problemas (problem stream); o fluxo das soluções (policy stream) e o fluxo político (politics stream). A tomada de decisão se dá com a junção desses fluxos por intermédio de uma "janela de oportunidade" (policy window) que se abre por uma "conjuntura temporal" e pela ação do empreendedor político (policy entrepreneur). Essa junção pode provocar mudanças, as vezes drásticas, na atenção dos tomadores de decisão e nas políticas públicas, de acordo com as habilidades do empreendedor em questão. ${ }^{3}$

O fluxo de problemas possui informações sobre variadas questões e por sujeitos que propõem definições complexas para esses problemas. A análise do fluxo se dá pela identificação e descrição dos mecanismos (indicadores, crises, eventos focalizadores e feedback de ações).

O fluxo das soluções é também conhecido como fluxo de alternativas ou da política propriamente dita e corresponde ao processo de construção e escolha das alternativas. A maior parte desse processo ocorre dentro de comunidades de especialistas, que podem ser acadêmicos, burocratas ou outros especialistas em domínio específico. Essas comunidades podem estar fragmentadas em menor ou maior grau. Kingdon ${ }^{1}$ dá grande importância às ideias, às interpretações e às argumentações no processo de formulação de políticas públicas e defende que em alguns casos, ideias são mais importantes que a influência dos grupos de pressão no processo decisório.

O fluxo político, independentemente do reconhecimento de um problema ou das alternativas disponíveis, é caracterizado pela barganha e formação de coalizão constrói consenso e compõe a agenda. Nesta perspectiva, três fatores podem influenciar o fluxo político: o clima nacional (national mood), forças políticas organizadas (como grupos de pressão) e as mudanças dentro do governo. O clima nacional refere-se a linhas comuns sobre as quais um número grande de pessoas converge caracterizado pelo compartilhamento de preocupações e percepções entre as pessoas. As forças políticas sinalizam aos formuladores se o ambiente é favorável ou não às propostas. $\mathrm{O}$ último componente do fluxo político é o governo, em que tanto mudanças no poder executivo, quanto no legislativo podem gerar importantes mudanças na agenda de políticas públicas.

Além dos três fluxos apresentados anteriormente (dos problemas, das soluções e político), o autor esclarece que há mais dois recursos estruturantes, que são: a janela de oportunidades e o empreendedorismo político. A janela de oportunidade se abre quando algum evento ou questão importante surge como uma oportunidade para os empreendedores políticos manipularem as informações a fim de apresentarem as soluções de que dispõe para determinado problema, ou seja é a combinação entre problemas-soluções-participantes-escolhas-alternativas. Assim a janela se apresenta como uma oportunidade tanto para gestores como para os empreendedores políticos, requerendo ação imediata por parte desses, uma vez que há considerações estratégicas e restrições lógicas.

Mudanças significativas nas políticas públicas ocorrem quando os três fluxos se juntam. Para 
que isso ocorra, é necessário que os empreendedores políticos aproveitem a oportunidade (policy windows). A figura do empreendedor de políticas oferece grande força explicativa frente a situações práticas de análise de políticas públicas já que, em tese, possuem capacidade para se fazerem ouvir, para fazerem conexões políticas e para negociarem, devendo estar sempre prontos para agir quando surge a janela, apresentando propostas e integrando fluxos.

\section{Trajetória metodológica do estudo}

Trata-se de estudo histórico-descritivo de natureza qualitativa. Histórico por levar em consideração uma herança cultural recebida, que vai construindo os momentos a partir de conformações e espaços de práticas. Descritivo porque possibilita obter uma visão geral do assunto pesquisado e tem como finalidade desenvolver, esclarecer e modificar ideias permitindo conhecer ou aumentar o conhecimento em torno de um dado problema possibilitando a proposição de estratégias de intervenções em determinadas situações ${ }^{4}$.

A opção pela abordagem qualitativa deve-se ao fato desta adequar-se ao estudo da configuração de processos particulares e específicos de grupos mais ou menos delimitados em extensão e capazes de serem englobados intensamente, aprofundando em sua complexidade ${ }^{5}$.

O processo de observação e pesquisa seguiu as seguintes etapas: (i) análise documental, realizada através de portarias ministeriais, editais, relatórios, seminários e oficinas realizada (artigos, livros, relatórios, outros); e (ii) análise dos dados oficiais oriundos da base de dados do Sistema de Informação da Atenção Básica /Departamento da Atenção Básica/DAB/Secretaria da Atenção à Saúde - SAS/Ministério da Saúde/MS.

Vale ressaltar que em consulta realizada na base de artigos Scielo organizada pela Bireme foram localizados 470.194 artigos dentre 1194 periódicos. Dentre as palavras-chave pesquisadas foram encontrados: 116 artigos sobre atenção básica, 458 sobre saúde da família; 33 sobre estratégia de saúde da família e 26 sobre formação da agenda e políticas públicas.

Para análise desses processos foram utilizadas as seguintes categorias: (1) fluxo dos problemas (problems stream); (2) fluxo de alternativas (policy stream); (3) fluxo político (political stream).

\section{Revisão da literatura: políticas públicas}

Este artigo destina-se a uma breve revisão da produção relativa aos campos teórico-práticos que dizem respeito às (aos): teorias de estado e de governo; conceitos de políticas públicas, políticas sociais, federalismo brasileiro; o modelo de análise dos múltiplos fluxos; as conexões histórico-políticas dos atores envolvidos no processo de construção da agenda da atenção básica/ estratégia de saúde da família e principalmente a apresentação dos principais fluxos que envolvem os momentos históricos. 
A Teoria Geral do Estado investiga e expõe os princípios fundamentais da sociedade política, sua origem, estrutura, formas, finalidade e evolução. Ao tratamento dos temas do Estado ou a ele relacionados dá-se o nome de política. O Estado e a política têm em comum a referência ao poder. Não há teoria política que não parta, direta ou indiretamente, de uma definição de poder e de uma análise do fenômeno do poder. Assim, é no poder político, a princípio aquele que tem exclusividade do uso da força, mas não necessariamente a física, que se estabelece mais eficazmente essa relação.

A teoria do Estado apoia-se, a partir dessa premissa, na teoria dos três poderes - o legislativo, o executivo e o judiciário - e na relação entre eles. É a inserção da teoria do Estado como uma parte da teoria política e desta, por sua vez, como parte da teoria do poder. "Por longa tradição o Estado é definido como o portador da summa potestas: e a análise do Estado se resolve quase totalmente no estudo dos diversos poderes que competem ao soberano" " ${ }^{6}$. Se as teorias do Estado e da política derivam da teoria (ou das teorias) do poder, é nela, a priori, que se deve pensar.

Para se adotar uma compreensão sintética compatível com os objetivos deste trabalho, o Estado pode ser considerado o conjunto de instituições permanentes - como órgãos legislativos, tribunais, exército e outras que não formam um bloco monolítico necessariamente - que possibilitam a ação do governo; e Governo, como o conjunto de programas e projetos que parte da sociedade (políticos, técnicos, organismos da sociedade civil e outros) propõe para a sociedade como um todo, configurando-se a orientação política de um determinado governo que assume e desempenha as funções de Estado por um determinado período.

As políticas públicas são aqui compreendidas como as de responsabilidade do Estado - quanto à implementação e manutenção a partir de um processo de tomada de decisões que envolve órgãos públicos e diferentes organismos e agentes da sociedade relacionados à política implementada. Neste sentido, políticas públicas não podem ser reduzidas a políticas estatais, mas como o "Estado em ação" em que o Estado age implantando um projeto de governo, através de programas, de ações voltadas para setores específicos da sociedade.

No Dicionário de Política ${ }^{7}$, a política pública é definida como um conjunto de disposições, medidas e procedimentos que traduzem a orientação política do Estado e regulam as atividades governamentais relacionadas às tarefas de interesse público, atuando e influindo sobre as realidades econômicas, social e ambiental.

Kingdon ${ }^{1}$ conceitua política pública como um conjunto de quatro processos: definição da agenda; especificação das alternativas; tomada de decisão e implementação da decisão.

Embora não haja consenso quanto à definição do que seja uma política pública, a definição mais conhecida continua sendo a de Laswell ${ }^{8}$ que defende que decisões e análises sobre política pública implicam responder às seguintes questões: quem ganha o quê, por quê e que diferença faz.

A questão social surge como resposta aos conflitos surgidos entre o capital e trabalho e como 
enfrentamento das desigualdades oriundas da exploração das sociedades capitalistas com o desenvolvimento da industrialização.

O termo política social, no âmbito das Ciências Sociais, é entendida como modalidade de política pública e, pois, como ação de governo com objetivos específicos. Como política pública, portanto, a política social deve ser entendida em sua dimensão política e histórica. E é contemplando estas dimensões, sempre articuladas, que se pode avançar um pouco mais na definição de política social e na identificação de seu objeto.

Laurell ${ }^{9}$ descreve as políticas sociais como o conjunto de medidas e instituições que tem por objetivo o bem-estar e os serviços sociais, para ampliar a cidadania, a inclusão social e política dos indivíduos. No entanto, em uma sociedade desigual, o Estado tem um papel redistributivo, desenvolvendo políticas do ponto de vista econômico com caráter compensatório e promocional para oferecer serviços à população e controlar o modo de relações na sociedade.

Conforme entendimento de Viana ${ }^{10}$, se política pública é ação governamental com objetivos específicos, política social é ação governamental com objetivos específicos relacionados com a proteção social.

Tomando como base a teoria do Estado federal moderno, define-se federalismo como uma forma particular de organização político-territorial do poder do Estado que reparte a autoridade política do Estado (a soberania) e o exercício do poder (o governo) em múltiplos centros soberanos definidos geograficamente e coordenados entre si.

Elazar ${ }^{11}$ enfatiza que, na ciência política, a divisão de poder é normalmente utilizada como sinônimo de federalismo. E por antecedência, são as instituições federativas compostas por suas estruturas, regras e processos que desenham a federação.

No Brasil, que vivencia ao longo de sua história mudanças importantes em relação a seus arranjos federativos e onde suas instituições sobrevivem mesmo após longos períodos de autoritarismo e ditadura militar, o federalismo desponta como solução para amortecer as enormes disparidades regionais.

Outra consideração a ser feita com relação ao federalismo é que as instituições federativas guardam estreita relação com os processos de descentralização. Abrucio ${ }^{12}$ conceitua descentralização como um processo político circunscrito a um Estado Nacional envolvendo diferentes instâncias de governo e por vezes, decorrente da conquista ou transferência de poder e governos subnacionais.

Em contextos federativos, a descentralização interfere no equilíbrio entre a autonomia e interdependência dos governos, e por isso, geralmente necessita do fortalecimento de mecanismos de coordenação que não se resumem à imposição de formas de participação conjunta, mas sim, da instauração de parcerias que sejam aprovadas pelos entes federativos. Desse modo, a 
descentralização é bem mais complexa, pois envolve jogos de cooperação e competição, acordos, vetos e decisões conjuntas entre níveis de governo ${ }^{12}$.

Arretche ${ }^{13}$ argumenta em seus estudos sobre federalismo que a dispersão da autoridade política em múltiplos centros potencializa o poder de veto das minorias e dificulta o processo de implantação de uma política. Relata ainda que com as mudanças nas instituições federativas a partir da Constituição de 1988, o governo federal encontra dificuldades para aprovar e executar sua agenda de reformas, devido à arquitetura institucional brasileira "descentralizada, estadualista e incompleta" e à indefinição e superposição de competências e responsabilidades associadas à descentralização fiscal.

Autores ${ }^{12,13}$ destacam a ausência de mecanismos cooperativos mais eficazes, quer seja entre a União e estados, quer seja na relação entre eles, o que leva a uma situação de "federalismo predatório" marcada pelo perpétuo conflito em torno dos recursos a serem atribuídos a cada esfera de governo, o que impede a implantação de políticas mais eficazes.

Viana e Oliveira ${ }^{14}$ destacam ainda que o processo de descentralização de políticas sociais ainda é lento e negociado, pois supõe o entendimento entre autoridades dotadas de graus diferenciados de legitimidade, autonomia decisória e recursos, desenvolvimento de mecanismos que fortaleçam a capacidade de cooperação e integração regional adequado delineamento de competência em função de um determinado modelo de intervenção econômica e social.

O tema da descentralização está bastante presente na literatura das políticas públicas sociais, sobretudo no que se refere à saúde. Em parte isso se deve à prioridade que a descentralização ganhou na agenda política dos anos 90 e à consequente feição descentralizada com que a área social vem ganhando no Brasil. Nas últimas duas décadas, a descentralização alterou o papel federal na política, ao mesmo tempo em que reafirmou a relevância do Ministério da Saúde na definição de políticas, no financiamento e na regulação do sistema de saúde.

A singularidade de um projeto de política social concebido com base no modelo federalista e respeitando a lógica participativa (movimentos sociais, sociedade civil) acrescentou algumas características importantes ao sistema brasileiro. Esse novo modelo estaria assentado na separação entre financiamento a cargo das três esferas de governo e a provisão dos serviços sob a responsabilidade dos municípios. A municipalização da gestão dos serviços foi o elemento central da agenda de reformas do governo federal na área da saúde na década de 1990.

É então fundamental produzir uma reflexão que procure compreender a articulação entre os formuladores de políticas e os sujeitos políticos que efetivamente decidem e implementam a "institucionalização" de determinadas ações do aparato público, tendo em vista que é neste universo que se tem colocado o conjunto das relações dos agentes sociais.

Nessa perspectiva, foi realizada uma análise histórica da ESF na tentativa de compreender 
como sua agenda foi formada.

\section{Análise histórica da estratégia de saúde da família em momentos históricos e fluxos}

A ESF foi instituída em março de 1994, mas somente em 1997 que as normas e diretrizes foram aprovadas por meio da Portaria $\mathrm{n}^{\mathrm{o}} 1.886{ }^{15}$. Ainda no sentido de acompanhar a trajetória histórica da ESF e de seu fortalecimento, houve a estratégia operacional dos Pólos de Formação, Capacitação e Educação Permanente (2002) ${ }^{16}$, o Projeto de Expansão e Consolidação do Saúde da Família - PROESF (2003) ${ }^{17}$, a Avaliação da Melhoria da Qualidade - AMQ (2004) ${ }^{18}$, a Norma Operacional Básica - NOB $2006{ }^{19}$ a definição do piso de atenção básica - PAB (2008) ${ }^{20}$, a Política Nacional da Atenção Básica - PNAB (2006) ${ }^{21}$ e sua revisão (2011) ${ }^{22}$ até o estabelecimento do Programa Nacional de Melhoria do Acesso e da Qualidade da Atenção Básica (PMAQ) ${ }^{23}$ em 2011.

O primeiro momento para a análise do processo de formação da agenda da Estratégia de Saúde da Família é a análise do Programa de Saúde da Família (PSF), concebido como uma estratégia prioritária da atenção básica para reorientação das práticas convencionais de atenção.

A expansão da ESF começou em áreas pobres e com pouca disponibilidade de serviços de saúde e cresceu rapidamente de 1998 e 2013, aumentando o número de equipes de 4.000 para mais de 34.000. No entanto, de 2002 a 2013, embora tenha crescido lentamente, o número de equipes do PSF teve um crescimento superior a cem por cento.

Ao longo de dez anos, o PSF demonstra evolução com relação à cobertura populacional. Atualmente a ESF abrange cerca de $60 \%$ da população brasileira, representando em números absolutos 5.328 municípios atendidos, o que representa $95,6 \%$ de cobertura.

Ao longo dos anos 2000 o Brasil experimentou uma forte redução da taxa de mortalidade infantil, esse decréscimo foi proporcionalmente maior no nordeste do Brasil. A literatura demográfica, que se dedica a estudar essas questões, aponta como determinantes do aumento da expectativa ao nascer, além das variáveis socioeconômicas da família, fatores relacionados às condições de saneamento do domicílio, identificam também a importância do efeito dos programas de saúde com foco nas famílias e comunidades, sobre a saúde das crianças ${ }^{24,25}$.

Com relação a este primeiro momento de análise, constitui-se como fluxo de problemas deste primeiro momento: a crise estrutural do setor público que se apresenta pela ineficiência do setor saúde; e se confirma pela insatisfação da população com os serviços de saúde e pela desqualificação profissional. Além destes, verifica-se, ainda, dificuldades de acesso aos serviços de saúde; alta taxa de mortalidade infantil causadas por diarreia, desnutrição e infecção respiratória aguda; hegemonia dos modelos de atenção médico-assistencial, privatista e sanitarista; coexistência de doenças crônico-degenerativas e infecciosas/parasitárias; recrudescimento de endemias como a dengue, tuberculose, malária e hanseníase 
Como fluxo de soluções, o PSF traz uma aproximação da equipe com a comunidade criando espaços de reconhecimento mútuo priorizando a qualidade de vida das pessoas. Ao se dirigir às famílias e ao seu meio ambiente, espera-se que as equipes realizem uma ação contínua personalizada e ativa, com base no estabelecimento de vínculos e criação de compromisso e corresponsabilidade entre os profissionais de saúde.

No que diz respeito ao fluxo político deste primeiro momento de análise: o Programa Saúde da Família (1994 a 1996) pode ser considerado como resposta a uma demanda de secretários municipais de saúde que queriam apoio para efetuar mudanças na forma de operações da rede básica de saúde através da expansão do Programa de Agentes Comunitários de Saúde (PACS) para outros tipos de profissionais.

O segundo momento ocorre a partir de 1996, quando ocorreu um avanço no estabelecimento de sistemáticas de financiamento das ações de atenção básica a partir do processo de descentralização. Nesta época são implementadas medidas de incentivo, expressas principalmente, na NOB/96 ${ }^{19}$ para que os municípios pudessem assumir progressivamente a gestão local da rede de serviços de saúde, recebendo recursos diretamente do governo central como forma de incentivar a responsabilidade de gerir suas próprias redes, e promover mudanças nas condições de acesso e na qualidade do atendimento prestado na rede pública.

A NOB/96 foi criada através da Portaria GM/MS no $1882{ }^{26}$ de 1997 e disciplinou o processo de organização dos serviços segundo campos de atenção assistenciais, de intervenções ambientais e de políticas extra-setoriais, em uma concepção ampliada de atenção á saúde e de integralidade de atenções, alavancando o processo descentralizador e possibilitando a concretização da principal estratégia de financiamento da atenção básica, a partir de 1998, com a introdução de um novo modelo de transferência de recursos financeiros: o PAB (Piso de Atenção Básica).

A NOB/96 teve entre suas principais determinações: (1) substituir o modelo de alocação de recursos financeiros federais para estados e municípios até então com base em convênios e pagamento por produção de serviços realizados pelo Fundo Nacional de Saúde; (2) fortalecer a capacidade gestora do Estado em nível local, incentivando mudanças no modelo de atenção básica por meio da ESF e (3) definir indicadores de produção e de impacto epidemiológico ${ }^{27}$.

O PAB altera a lógica de repasse de recursos federais para o sistema de prestação de ações médico-sanitárias e se constitui uma medida decisiva na perspectiva da descentralização financeira do sistema de saúde no Brasil, incentivando os municípios a assumirem, progressivamente, a gestão da rede básica de serviços de saúde.

O PAB se constitui em um componente federal, para o financiamento da Atenção Básica, sendo composto por uma fração fixa e outra variável destinadas a viabilização das ações de $\mathrm{AB}$ alterando a lógica de financiamento da assistência à saúde, dissociando produção e faturamento trazendo como 
principal novidade a transferência regular e automática com repasse federal feito diretamente aos municípios.

A condição de habilitação ao PAB definida pela NOB/96 obriga a criação do Fundo Municipal de Saúde e do Conselho Municipal de Saúde, dando relativa autonomia e crescente responsabilidade ao gestor municipal. Esse novo mecanismo dissocia a produção do faturamento, característica central do sistema anterior.

A lógica do pós-pagamento, considerada parte do fluxo de problemas deste momento, impressa no antigo sistema de pagamentos estava prejudicando os municípios mais carentes quando comparados aos municípios mais desenvolvidos, pois recebiam valores menores devido a precariedade de suas redes de saúde, enquanto os maiores por apresentarem um número maior de hospitais, ambulatórios e postos de saúde, recebiam valores maiores incentivando a lógica de superfaturamento.

Há quem afirme que a NOB/96, em que pese ser um instrumento na operacionalização da descentralização do sistema, ao incrementar transferências diretas fundo a fundo no campo da Atenção Básica, pode impedir ou obstaculizar a construção de uma política de saúde fundada nas necessidades locais. Bueno e Merhy ${ }^{28}$ afirmam que a NOB/96 poderia inibir a autonomia do município, induzindo-o a adotar programas não definidos localmente.

Elias, Marques e Mendes ${ }^{29}$ reforçam que o processo de descentralização não foi acompanhado pela autonomia dos municípios na determinação da política de saúde. Esses, dependentes dos recursos federais, passaram a ser meros executores da política estabelecida no âmbito federal, o que torna a participação da comunidade princípios cada vez mais esmaecidos em nome da eficiência do gasto em saúde.

Segundo Paim et al. ${ }^{30}$ esse novo acordo federativo deu mais autonomia aos municípios, mas também expandiu os recursos e controles do nível federal. A saúde foi o único setor que implementou uma descentralização radical, com importante financiamento e ações regulatórias do governo federal.

O fato de a descentralização ter sido assumida como um mecanismo que por si só traria consigo a democratização da gestão da saúde, porque prevê a participação da sociedade na gestão dos sistemas e dos serviços de saúde e, consequentemente, maior adequação das políticas locais às necessidades de saúde da população, esse processo vem se dando de forma bastante diferenciada e questionadora, portanto, com a premissa de que a descentralização seja sinônimo de democratização.

O terceiro momento identificado neste estudo foi a criação dos Pólos de Capacitação, Formação e Educação Permanente de Pessoal para a Saúde da Família em 2002. Com o aumento do número de equipes de saúde da família desde 1997 foi necessário contratar mais profissionais de saúde em todo o território nacional, bem como a construção ou reestruturação das unidades de saúde. 
No entanto, os profissionais com conhecimentos, habilidades e atitudes adequados ao novo modelo proposto pela ESF não estavam disponíveis no mercado de trabalho porque tinham formação generalista e eram preparados para atuarem no modelo tradicional. O perfil inadequado dos profissionais passou a ser o grande obstáculo para a consolidação da estratégia.

Para o enfrentamento dessa realidade, em 1996, o Ministério da Saúde propôs aos serviços e as instituições de ensino o estabelecimento de parcerias para que o processo de capacitação das equipes de Saúde da Família pudesse ser concretizado e fosse ainda possível influenciar no processo de formação desses profissionais. Assim, foram criados os Pólos de Capacitação, Formação e Educação Permanente para o $\mathrm{PSF}^{31}$, conhecidos também como rodas de gestão locorregionais entendidas como espaços para o estabelecimento do diálogo e da negociação entre as ações e os serviços do SUS e as instituições de ensino com o objetivo de preparar os profissionais que iriam compor as equipes da ESF no Brasil.

A Educação Permanente definida como ação pedagógica com foco no cotidiano do trabalho em saúde vem dar sustentação à criação dos Pólos já que, de acordo com Merhy ${ }^{32}$, a proposta de Educação Permanente requer o desenvolvimento de ações em diversos âmbitos: da formação técnica, de graduação e de pós-graduação; da organização do trabalho; da interação com as redes de gestão e de serviços de saúde; e do controle social neste setor.

Em 2004, a Política Nacional de Educação Permanente em Saúde foi instituída como estratégia de transformação do SUS por meio da articulação entre saúde e educação. Essas duas áreas/temáticas citadas como prioridade representaram $40 \%$ do total de ações de qualificação (desenvolvimento profissional e institucional) e $40 \%$ das ações de especialização (formação pós-graduada de lato sensu) apresentadas para o apoio do Ministério da Saúde, em 2004, por meio dos Polos de Educação Permanente em Saúde.

Ao propor a educação na saúde como política de governo, o gestor federal do SUS alterou uma das prioridades no setor somando novos atores. Colocar a Educação Permanente em Saúde como prioridade para o SUS evidenciou o trabalho da saúde, um trabalho que requer: trabalhadores que aprendam a aprender; práticas cuidadoras; intensa permeabilidade ao controle social; compromissos de gestão com a integralidade; desenvolvimento de si, dos coletivos, institucional e político da saúde, além da implicação com as práticas concretas de cuidado às pessoas e às coletividades, no ensino e na produção de conhecimento.

Em 2002, a ESF ampliou e passou a ser apoiada pelo o Projeto de Expansão e Consolidação Saúde da Família (PROESF), estratégia negociada pelo Ministério da Saúde financiada pelo Banco Mundial para organizar e fortalecer a Atenção Básica à Saúde no País em municípios com população acima de 100 mil habitantes. Este se constituiu o quarto momento de análise da agenda da Estratégia de Saúde da Família. 
Além de contribuir para a implantação e consolidação da Estratégia de Saúde da Família em municípios de grande porte, o PROESF objetiva a elevação da qualificação do processo de trabalho e desempenho dos serviços, por meio da ampliação e reestruturação das várias iniciativas voltadas para o desenvolvimento de recursos humanos no PSF para elevar a qualificação do processo de trabalho e desempenho dos serviços em todos os municípios brasileiros, otimizando e assegurando respostas efetivas para a população.

O quinto momento incorpora a questão da avaliação em saúde, esta ainda incipiente e pouco incorporada às práticas de gestão. Os instrumentos existentes ainda não se constituem ferramentas de suporte ao processo decisório nem da formação de pessoas constituindo parte do fluxo de problemas. Goulart ${ }^{33}$ aponta que a prática de avaliação existente se expressa mais intensamente no Ministério da Saúde a partir de 1999 e 2000, tendo o PSF como principal indutor.

Nesse contexto a Avaliação para a Melhoria da Qualidade da Estratégia de Saúde da Família (AMQ) surge como uma janela de oportunidade e com o objetivo geral de fomentar a avaliação de dos estágios de qualidade da ESF aproximando os atores envolvidos na ESF dos métodos e instrumentos para a autoavaliação de maneira a auxiliar no processo de tomada de decisão ${ }^{34}$.

A AMQ representa uma metodologia de autoavaliação e gestão interna da qualidade, com vistas ao desenvolvimento de processos de melhoria contínua da qualidade. Traz o diferencial de oferecer aos gestores municipais, coordenadores e profissionais, a possibilidade de identificar pontos críticos, acompanhar o processo de implantação e desenvolvimento da ESF de maneira incremental e permanente.

Ao se autoavaliar, a AMQ reforçou, em cada membro da equipe, gestor ou profissional de saúde, a capacidade de reflexão sobre o seu processo de trabalho, a sua equipe, a diversidade e a integração do serviço com a comunidade reforçando o caráter coparticipativo e democrático, ao construir estratégias e ações comuns, com divisão de tarefas e responsabilidades, fazendo com que os resultados esperados fossem alcançados com maior facilidade.

O sexto momento refere-se à consolidação da Política Nacional de Saúde por meio da Portaria GM/MS no $650{ }^{35}$, de 28 de março de 2006 que estabeleceu a revisão de diretrizes e normas para a organização da Atenção Básica para o Programa Saúde da Família (PSF) e para o Programa Agentes Comunitários de Saúde (PACS), que foi revisada em 2011 por meio da Portaria $\mathrm{n}^{\circ} 2488$ ${ }^{36}$ de 21 de outubro de $2011^{37}$.

As principais alterações foram: incorporação de diretrizes e normas relativas a outras formas de organização da atenção básica, indo além da estratégia de Saúde da Família; incorporação de políticas voltadas para grupos populacionais específicos; inclusão das estratégias de promoção da atividade física e mudanças no financiamento.

O sétimo e último momento de análise deste estudo retoma a questão da avaliação dos sistemas 
de saúde nos três níveis de governo, a partir da institucionalização de uma estratégia indutora para implantação de processos que ampliem a capacidade das gestões federal, estaduais e municipais: o Programa Nacional de Melhoria do Acesso e da Qualidade da Atenção Básica (PMAQ-AB) ${ }^{23}$.

O PMAQ-AB, organizado em quatro fases cíclicas, visou impulsionar o processo de trabalho das equipes de atenção básica, a fim de ofertarem serviços que assegurem maior acesso e qualidade, de acordo com as necessidades concretas da população.

Dentre os desafios do PMAQ, está o enfrentamento para qualificação da atenção básica, destacam-se a precariedade da rede física; a ambiência pouco acolhedora das UBS; as inadequadas condições de trabalho para os profissionais; a necessidade de qualificação do trabalho das equipes; a instabilidade das equipes e a elevada rotatividade dos profissionais; a insipiência dos processos de gestão; a sobrecarga das equipes com número excessivo de pessoas sob sua responsabilidade; a pouca integração das equipes com a rede de apoio diagnóstico e terapêutico; a baixa integralidade e resolutividade das práticas e o financiamento insuficiente e inadequado ${ }^{38}$.

\section{CONSIDERAÇÕES FINAIS}

Considerando que o processo de formação de uma agenda governamental não acontece por acaso e que se julgou importante conhecer as estratégias adotadas pelo Ministério da Saúde, no âmbito da Atenção Básica, pelo fato de que possam ter influenciado a formação da agenda governamental da Estratégia de Saúde da Família, cabe destacar sete momentos considerados marcos históricos e prioritários para a Saúde da Família. Dos momentos de análise identificados neste trabalho, o primeiro deles foi o Programa de Saúde da Família - PSF, que pode ser considerado um modo inovador de se trabalhar saúde, à medida que "desafoga" a assistência curativa dos hospitais, diminuindo a demanda desnecessária sobre os especialistas, melhorando assim, o acesso de todos os usuários a procedimento que requeiram maior complexidade.

Como fluxo político, destaca-se que a ESF surge em um cenário de crise da capacidade do poder público de continuar financiando a expansão do modelo hospitalar privado, na contramão de uma sensibilidade sociopolítica, nacional e estadual, vinculada a projetos democráticos de reforma do Estado e a projetos universais interdisciplinares, intersetoriais, equitativos.

O financiamento em saúde, tema de que trata o segundo momento analisado neste trabalho, diz respeito a um conjunto de responsabilidades e prerrogativas de gestão para o nível local. A NOB 96 veio romper com o mecanismo de repasse baseado em produção de serviços e foi uma resposta a necessidade de descentralização de recursos da Atenção Básica. Do mesmo modo, foi criado o Piso da Atenção Básica (PAB), sendo composto de uma parte fixa, associado a lógica de financiamento per capita e outra variável, em que os municípios passam a receber recursos diretamente do governo federal assumindo a responsabilidade e capacidade de ofertar ações e dos serviços de saúde.

Como a análise dos momentos, não segue necessariamente uma ordem cronológica, o terceiro 
momento está situado entre os dois primeiros e surge em função da necessidade de se formar profissionais que pudessem desenvolver práticas mais consoantes com as propostas de Saúde da Família.

A compreensão integral do segmento familiar requer uma atitude compreensiva dos profissionais de saúde com relação a realidade de cada local, que está relacionada com fatores culturais e intrínsecos de cada família, além do entendimento de seus membros enquanto indivíduos.

A expansão da ESF e o impasse relacionado com a formação dos profissionais possibilitou a formação de novas alianças e articulações no cenário da política de saúde. Como solução a este impasse, surgiram os Polos de Formação, Capacitação e Educação Permanente estabelecidos a partir de 1998 para estimular mudanças curriculares no sistema formador de acordo com a lógica da promoção da saúde em uma perspectiva de prevenção. Para isso, foi necessário capacitar os profissionais de modo a prepará-los para a construção do cuidado familiar no intuito de se prestar uma assistência integral que atenda as necessidades de saúde e doença dos indivíduos.

O quarto momento corresponde à expansão da ESF para os grandes centros urbanos, a partir de 2003, com o Projeto de Expansão e Consolidação da Saúde da Família - PROESF. Esta iniciativa ocorreu devido à grande concentração populacional em grandes centros urbanos e às baixas coberturas apresentadas pelo PSF. Apesar do investimento de U\$ 550 milhões e do apoio do Banco Mundial, não foram encontradas informações sistematizadas sobre a execução financeira deste orçamento senão pelo Relatório de Avaliação do Projeto elaborado por este organismo internacional. Além disso, recebe críticas em relação a sua aplicabilidade em municípios maiores pelo fato de já existirem serviços consolidados ou outros processos em curso que dificultam a execução dessa proposta.

A Avaliação para a Melhoria da Qualidade (AMQ) da Estratégia de Saúde da Família, quinto momento analisado, surge com o objetivo de fomentar a avaliação dos estágios de qualidade da ESF trazendo a auto avaliação para os atores envolvidos como uma possibilidade de melhoria contínua da gestão. Integra também o componente III do PROESF que está relacionado à estruturação e implementação de metodologias e instrumentos de monitoramento e avaliação. Apesar de ser uma metodologia incipiente traz um aspecto formador para os atores envolvidos.

A consolidação e aprovação da Política Nacional de Atenção Básica em 2006 constitui-se o sexto momento deste trabalho, e foi apresentado como um marco histórico da política quando foi estabelecida a revisão de diretrizes e normas para a organização da Atenção Básica tanto para o PSF quanto para o PACS. Em 2011, a PNAB foi revista novamente para incluir mudanças que ocorreram desde então, por meio da consolidação do NASF, das equipes de SF ribeirinhas, do Programa Saúde na Escola e da flexibilização da carga horária médica nas ESF.

O sétimo e último momento de análise é o Programa Nacional de Melhoria do Acesso e da Qualidade da Atenção Básica que traz o desafio de qualificar a atenção básica por meio de um 
modelo de avaliação de desempenho dos sistemas de saúde nos três níveis de governo, vinculando parte dos recursos a serem repassados a padrões de qualidade que indicariam a ampliação do acesso aos serviços, a melhoria das condições de trabalho e da qualidade da atenção, o que representa uma mudança na lógica de repasse de recursos para a atenção básica e anuncia alterações na lógica de financiamento.

Este trabalho não teve como objetivo destrinchar a política da Atenção Básica á Saúde, mas compreender como ocorre o funcionamento do processo de formulação da agenda da ESF. Nesse sentido, o estudo cumpre seu objetivo, destacando que a ESF tem auxiliado o processo de gestão do SUS no que diz respeito à atenção básica, se configurando uma política .democratizante.

Apesar de todos os avanços que a Política Nacional de Atenção Básica alcançou no que diz respeito a ESF, enquanto estratégia de inversão do modelo assistencial por meio do trabalho de equipes e da ampliação do conceito de saúde para além das práticas curativas, considera-se que ainda existem alguns desafios a serem enfrentados para garantir e promover a ampliação do acesso do usuário.

Das críticas mais contundentes encontradas durante a realização deste estudo, está o fato da ESF ser definida como uma estratégia de reforma dos modos de atenção que ainda não conseguiu romper com o sistema tradicional predominante. Está voltado para a camada da população menos favorecida e não alcança a classe mais favorecida, incentivando dessa forma, a permanência e vitalidade do modelo tradicional. Além disso, é mister articular a demanda espontânea com a demanda programada; articular este nível de atenção com os demais; enfrentar a formação de recursos humanos; implantar efetivamente instrumentos de gestão para que se possa avaliar e acompanhar efetivamente esta Política prioritária de Saúde.

Entendemos que a conversão do modelo médico hegemônico para um modelo usuário centrado exige o enfrentamento de desafios colocados pela complexidade de sua operacionalização, que vão além dos identificados ao longo dos momentos trabalhados neste estudo, tais como: perfil epidemiológico, a questão do financiamento, os problemas de acesso, gestão e organização dos serviços de saúde.

Agradecimentos: Agradecimento à A.V.M. Mendonça, M.P.A. Zaitune e M.F. de Sousa que contribuíram na revisão e finalização do artigo.

\section{REFERÊNCIAS BIBLIOGRÁFICAS}

1. Kingdon JW. Agendas Alternatives and Public Policies. New York: HarperCollins; 1995.

2. Cohen MD; March JG; Olsen JP. A garbage can model of organizational choice. Adm sci $q$ $1972 ; 17(1): 1-25$. 
3. Pedroso M. Racionalidade limitada e uso de informações técnicas em modelos de análise de políticas públicas: proposições sobre a perspectiva integradora da Análise Multicritério de Decisão Espacial Construtivista. Rev Pesq Pol Pub 2013; 2: 59-83.

4. Triviños ANS. Introdução à Pesquisa em Ciências Sociais: a pesquisa qualitativa em educação. São Paulo: Atlas; 1992.

5. Minayo MCS; Sanches O. Quantitativo-qualitativo: oposição ou complementaridade. Cad Saude Publica 1993; 9(3): 239-262.

6. Bobbio N. Estado governo e sociedade: para uma teoria geral da política. Tradução de Marco Aurélio Nogueira. São Paulo: Paz e Terra; 2000.

7. Bobbio N; Matteusci N; Pasquino G. Dicionário de política. Brasília: Ed. UnB; 1991.

8. Lasswell H. Politics: Who gets what when how? Cleveland, OH: Meridian Books; 1936.

9. Laurell AC; Noriega M. Para o Estudo de Saúde na sua Relação com o Processo de Produção. In: Processo de Produção e Saúde: trabalho e desgaste operário. São Paulo: Hucitec; 1989.

10. Viana AL. Abordagens metodológicas em políticas públicas. Rev Adm Pública 2013; 30(2): $5-43$.

11. Elazar DJ. Exploring Federalism. The University of Alabama Press, 1987.

12. Abrucio FL. Descentralização e pacto federativo. Cad ENAP 1993;1(1): 17-33.

13. Arretche MTS. Políticas sociais no Brasil: descentralização em um estado federativo. Rev Bras Cienc Soc. 1999; 14(40): 111-41.

14. Viana AA.; Lima LD.; Oliveira RG. Descentralização e federalismo: a política de saúde em novo contexto-lições do caso brasileiro. Ciência Saúde Coletiva, 2002; 7(3), 493-507.

15. Brasil. Ministério da Saúde. Portaria ${ }^{0} 1.886$, de 22 de dezembro de 1997. Aprova as normas e diretrizes do Programa de Agentes Comunitários de Saúde e do Programa Saúde da Família. Diário Oficial da União 1997 dez 22; Seção 1. p. 11-13.

16. . Ministério da Saúde. Secretaria de Políticas de Saúde. Departamento de Atenção Básica. Reunião dos Coordenadores dos pólos de capacitação formação e educação permanente em saúde da família: relatório final. Brasília: Ministério da Saúde; 2002. 
17. Ministério da Saúde. Projeto de Expansão e Consolidação do Saúde da Família Proesf; 2003. [acesso em 2015 jul 30]. Disponível em http://bvsms.saude.gov.br/bvs/publicacoes/ PROESF.pdf

18. Ministério da Saúde. Secretaria de Atenção à Saúde. Departamento de Atenção Básica. Avaliação para melhoria da qualidade da estratégia saúde da família. Brasília: Ministério da Saúde; 2005. [acesso em 2015 jul 30]. Disponível em http://bvsms.saude.gov.br/bvs/ publicacoes/10001019925.pdf

19. . Ministério do Desenvolvimento Social e Combate à Fome. Norma Operacional Básica de Recursos Humanos NOB/RH. Brasília; 2006.

20. . Ministério da Saúde. Portaria $n^{0} 2.489$, de 21 de outubro de 2008. Define valores de financiamento do Piso da Atenção Básica Variável para a estratégia de Saúde da Família e de Saúde Bucal, instituídos pela Política Nacional de Atenção Básica. Diário Oficial da União. 2008 out. 21; Seção 1. p. 61.

21. Ministério da Saúde. Secretaria de Atenção à Saúde. Departamento de Atenção Básica. Política Nacional de Atenção Básica. Brasília: Ministério da Saúde; 2006.

22. . Ministério da Saúde. Política Nacional de Atenção Básica. Brasília: Ministério da Saúde; 2012.

23. Ministério da Saúde. Portaria no 1.654 , de 19 de julho de 2011. Institui, no âmbito do Sistema Único de Saúde, o Programa Nacional de Melhoria do Acesso e da Qualidade da Atenção Básica (PMAQ-AB) e o Incentivo Financeiro do PMAQ-AB, denominado Componente de Qualidade do Piso de Atenção Básica Variável - PAB Variável. Diário Oficial da União. 2011 jul. 19; Seção 1. p. 79.

24. Halstead SB; Walsh JA; Warren KS. Good Health at Low Cost: Proceedings of a Conference held at the Ballagio Conference Center Bellagio, Italy, from 29 April to 3 May 1985. New York: Rockefeller Foundation; 1985.

25. Caldwell JC. Routes to Low Mortality in Poor Countries. Popul Dev Rev 1986; 12 (2): 171 220.

26. Brasil. Ministério da Saúde. Gabinete do Ministro. Portaria $n^{0} 1.882$, de 18 de dezembro de 1997. Estabelece o Piso da Atenção Básica - PAB e sua composição. Diário Oficial da União. 1997 dez. 18; Seção 1. p. 10.

27. LevcovitzE; Lima L; Machado C.Política de saúde nos anos 90: relações intergovernamentais 
e o papel das Normas Operacionais Básicas. Cienc saude colet 2001; 6(2): 269-293.

28. Bueno WS.; Merhy EE. Norma Operacional Básica e o modelo tecnoassistencial

em saúde, mimeo, Campinas, 1997.

29. Elias PE.; Marques RM.; Mendes A. O financiamento e a política de saúde. Revista USP, São Paulo. n.51, p. 16-27, setembro/novembro, 2001.

30. Paim JS. Saúde Política e Reforma Sanitária. Salvador: CEPS-ISC; 2002.

31. Souza HM. O PSF como indutor da institucionalização da avaliação na atenção básica. Rev Bras Saúde Fam, [S.1.], v. 2, n. 6, p. 10-15, 2002.

32. Merhy E.; Feuerweker LCM.; Ceccim R. Educación Permanente en Salud: una estratégia para intervenir en la micropolítica del trabajo en salud. Salud coletiva, v.2, n.2,p.147-60, 2006.

33. Goulart FDA. Experiências em saúde da família: cada caso é um caso. Rio de Janeiro: Escola Nacional de Saúde Pública, Fundação Oswaldo Cruz, 2002.

34. Brasil. Ministério da Saúde. Secretaria de Atenção à Saúde. Departamento de Atenção Básica. Avaliação para Melhoria da Qualidade da Estratégia Saúde da Família: documento técnico. Brasília; 2005.

35. . Ministério da Saúde. Gabinete do Ministro. Portaria n ${ }^{\circ}$ 650, de 28 de março de 2006. Define valores de financiamento do PAB fixo e variável mediante a revisão de diretrizes e normas para a organização da Atenção Básica, para a estratégia de Saúde da Família e para o Programa de Agentes Comunitários de Saúde, instituídos pela Política Nacional de Atenção Básica. Diário Oficial da União. 2006 mar. 29; Seção 1.

36. . Ministério da Saúde. Gabinete do Ministro. Portaria n $n^{\circ}$ 2.488, de 21 de outubro de 2011. Aprova a Política Nacional de Atenção Básica, estabelecendo a revisão de diretrizes e normas para a organização da Atenção Básica, para a Estratégia Saúde da Família (ESF) e o Programa de Agentes Comunitários de Saúde (PACS). Diário Oficial da União. 2011 out. 21; Seção 1. p. 48-55.

37. . Ministério da Saúde. Secretaria de Atenção à Saúde. Departamento de Atenção Básica. Documento Síntese para Avaliação Externa do Programa Nacional de Melhoria do Acesso e da Qualidade da Atenção Básica (PMAQ). Brasília, 2012. Disponível em: <http://189.28.128.100/ $\mathrm{dab} / \mathrm{docs} / \mathrm{sistemas} / \mathrm{pmaq} /$ Documento_Sintese_Avaliacao_Externa_2012_04_25.pdf $>$. Acesso em dez. 2015. 
$305 / /$

38. _ _ Ministério da Saúde. Secretaria Executiva. Departamento de Monitoramento e Avaliação o SUS. Programa de Avaliação para Qualificação do Sistema Único de Saúde. Brasília, 2011.

Artigo apresentado em 20-02-15

Artigo aprovado em 02-11-15

Artigo publicado no sistema em 22-12-15 